EPJ manuscript No.

(will be inserted by the editor)

\title{
A new way to perform partial wave decompositions of few-nucleon forces
}

\author{
J. Golak ${ }^{1}$, D. Rozpędzik ${ }^{1}$, R. Skibiński ${ }^{1}$, K. Topolnicki ${ }^{1}$, H. Witała ${ }^{1}$, W. Glöckle ${ }^{2}$, A. Nogga ${ }^{3,4}$, E. Epelbaum ${ }^{3,5}, \mathrm{H}$ \\ Kamada $^{6}$, Ch. Elster ${ }^{7}$, and I. Fachruddin ${ }^{8}$ \\ 1 M. Smoluchowski Institute of Physics, Jagiellonian University, PL-30059 Kraków, Poland \\ 2 Institut für Theoretische Physik II, Ruhr-Universität Bochum, D-44780 Bochum, Germany \\ 3 Forschungszentrum Jülich, Institut für Kernphysik (Theorie) and Jülich Center for Hadron Physics, D-52425 Jülich, Germany \\ 4 Forschungszentrum Jülich, Institute for Advanced Simulation, D-52425 Jülich, Germany \\ 5 Helmholtz-Institut für Strahlen- und Kernphysik (Theorie) and Bethe Center for Theoretical Physics, Universität Bonn, \\ D-53115 Bonn, Germany \\ 6 Department of Physics, Faculty of Engineering, Kyushu Institute of Technology, Kitakyushu 804-8550, Japan \\ 7 Institute of Nuclear and Particle Physics, Department of Physics and Astronomy, Ohio University, Athens, OH 45701, USA \\ 8 Departemen Fisika, Universitas Indonesia, Depok 16424, Indonesia
}

Received: date / Revised version: date

\begin{abstract}
We formulate a general and exact method of partial wave decomposition (PWD) of any nucleonnucleon $(\mathrm{NN})$ potential and any three-nucleon $(3 \mathrm{~N})$ force. The approach allows one to efficiently use symbolic algebra software to generate the interaction dependent part of the program code calculating the interaction. We demonstrate the feasibility of this approach for the one-boson exchange BonnB potential, a recent nucleon-nucleon chiral force and the chiral two-pion-exchange three-nucleon force. In all cases very good agreement between the new and the traditional PWD is found. The automated PWD offered by the new approach is of the utmost importance in view of future applications of numerous chiral N3LO contributions to the $3 \mathrm{~N}$ force in three nucleon calculations.
\end{abstract}

PACS. 21.45.-v few-body systems - 21.30.-x nuclear forces -21.45 .Bc two-nucleon system - 21.45.Ff three-nucleon forces

\section{Introduction}

The standard way to set up calculations of two- and threenucleon systems is a partial wave decomposition (PWD). Especially at low energies, i.e. below the pion production threshold, this procedure is still most commonly used, despite the advent of the approaches which use a direct three-dimensional notation [1,2,3,4].

Recently we proposed a formulation of the two- and three-nucleon system [5, 6, which is based on scalar spinmomentum operators and accompanying scalar functions depending only on the momenta of the system. This formulation is based on the most general operator structure a nuclear force given in momentum space can have. The two- and three-nucleon equations are obtained by carrying out traces over the spin-momentum operators building the nuclear force. The same approach can be used to obtain partial wave projected matrix elements of the potential and the transition operators. Taking traces of spinmomentum operators lends itself to the use of symbolic algebra software to obtain general expressions for those matrix elements.

In this work we demonstrate that by algebraic operations general expressions for the partial wave decompo- sition of any nucleon-nucleon $(\mathrm{NN})$ potential can be obtained. This method will be presented in Sec. 2 and explicit expressions for calculating specific matrix elements of NN potentials are given in an appendix. Numerical comparisons between our suggested methods and the standards partial wave decomposition using recent chiral NN forces $[7$ and the one-boson-exchange NN potential BonnB 8 are presented in Sec. 3. In Sec. 4 we demonstrate that the same method can be extended to the PWD of a threenucleon force. As a numerical example given in Sec. 5 we take the two-pion-exchange (TPE) chiral NNLO threenucleon force. Finally we conclude in Sec. 6]

\section{Partial wave decomposition of the NN potential}

We start, as in Ref. 6, by projecting the NN potential on the two-nucleon (2N) isospin states $\left|\left(\frac{1}{2} \frac{1}{2}\right) t m_{t}\right\rangle \equiv\left|t m_{t}\right\rangle$. Furthermore, we assume that there is no isospin mixing but allow for charge independence and charge symmetry breaking, and thus for a dependence on $m_{t}$ :

$$
\left\langle t^{\prime} m_{t}^{\prime}|V| t m_{t}\right\rangle=\delta_{t^{\prime} t} \delta_{m_{t}^{\prime} m_{t}} V^{t m_{t}} .
$$


It is well known that the most general form of the $\mathrm{NN}$ force, which is invariant under rotations, parity and time reversal can be expressed by six scalar spin-momentum operators [10, which we choose as

$$
\begin{aligned}
& w_{1}\left(\boldsymbol{\sigma}(1), \boldsymbol{\sigma}(2), \boldsymbol{p}^{\prime}, \boldsymbol{p}\right)=1, \\
& w_{2}\left(\boldsymbol{\sigma}(1), \boldsymbol{\sigma}(2), \boldsymbol{p}^{\prime}, \boldsymbol{p}\right)=\boldsymbol{\sigma}(1) \cdot \boldsymbol{\sigma}(2), \\
& w_{3}\left(\boldsymbol{\sigma}(1), \boldsymbol{\sigma}(2), \boldsymbol{p}^{\prime}, \boldsymbol{p}\right)=i(\boldsymbol{\sigma}(1)+\boldsymbol{\sigma}(2)) \cdot\left(\boldsymbol{p} \times \boldsymbol{p}^{\prime}\right), \\
& w_{4}\left(\boldsymbol{\sigma}(1), \boldsymbol{\sigma}(2), \boldsymbol{p}^{\prime}, \boldsymbol{p}\right)=\boldsymbol{\sigma}(1) \cdot\left(\boldsymbol{p} \times \boldsymbol{p}^{\prime}\right) \boldsymbol{\sigma}(2) \cdot\left(\boldsymbol{p} \times \boldsymbol{p}^{\prime}\right), \\
& w_{5}\left(\boldsymbol{\sigma}(1), \boldsymbol{\sigma}(2), \boldsymbol{p}^{\prime}, \boldsymbol{p}\right)=\boldsymbol{\sigma}(1) \cdot\left(\boldsymbol{p}^{\prime}+\boldsymbol{p}\right) \boldsymbol{\sigma}(2) \cdot\left(\boldsymbol{p}^{\prime}+\boldsymbol{p}\right), \\
& w_{6}\left(\boldsymbol{\sigma}(1), \boldsymbol{\sigma}(2), \boldsymbol{p}^{\prime}, \boldsymbol{p}\right)=\boldsymbol{\sigma}(1) \cdot\left(\boldsymbol{p}^{\prime}-\boldsymbol{p}\right) \boldsymbol{\sigma}(2) \cdot\left(\boldsymbol{p}^{\prime}-\boldsymbol{p}\right)
\end{aligned}
$$

Thus the isospin projected potential can be expresses as

$$
V^{t m_{t}}\left(\boldsymbol{p}^{\prime}, \boldsymbol{p}\right)=\sum_{i=1}^{6} f_{i}\left(\boldsymbol{p}^{\prime}, \boldsymbol{p}\right) w_{i}\left(\boldsymbol{\sigma}(1), \boldsymbol{\sigma}(2), \boldsymbol{p}^{\prime}, \boldsymbol{p}\right) .
$$

The expansion coefficient here are scalar functions $f_{i}\left(\boldsymbol{p}^{\prime}, \boldsymbol{p}\right)$ that depend on two vector momenta $\boldsymbol{p}^{\prime}$ and $\boldsymbol{p}$, more specifically on the magnitudes of the vectors and and the cosine of the relative angle between them. In order to determine the functions $f_{i}$, we evaluate the spin dependence analytically by taking traces with the operators of Eq. (2) and thus arrive at a system of six coupled linear equations

$$
\sum_{j=1}^{6} \operatorname{Tr}\left(w_{i} w_{j}\right) f_{i}=\operatorname{Tr}\left(V^{t m_{t}} w_{i}\right), \quad i=1,2, \ldots, 6,
$$

which has a unique solution provided that $p^{\prime} \neq p$. (When $p^{\prime}=p$ only five out of the six operators $w_{i}$ are sufficient, since $w_{2}$ is linearly dependent on $w_{4}, w_{5}$, and $w_{6}$.)

Our task is to obtain matrix elements of $V^{t m_{t}}$ in the basis of states $\left|p(l s) j m_{j}\right\rangle$, where the relative angular momentum $l$ and the total spin $s$ are coupled to the total angular momentum $j$ with its projection $m_{j}$. When calculating NN observables one usually sums angular momenta $j$ up to a certain $j_{\max }$ at which the calculation is converged. For calculations of NN observables below the pion production threshold convergence is reached for $j_{\max } \leq 8$.

To obtain the potential matrix element in the basis $\left|p(l s) j m_{j}\right\rangle$, the four-fold integral

$$
\begin{aligned}
& \left\langle p^{\prime}\left(l^{\prime} s\right) j m_{j}\left|V^{t m_{t}}\right| p(l s) j m_{j}\right\rangle= \\
& =\int d \hat{p}^{\prime} \int d \hat{p} \sum_{m_{l}^{\prime}} c\left(l^{\prime}, s, j ; m_{l}^{\prime}, m_{j}-m_{l}^{\prime}, m_{j}\right) \\
& \quad \times \sum_{m_{l}} c\left(l, s, j ; m_{l}, m_{j}-m_{l}, m_{j}\right) Y_{l^{\prime} m_{l}^{\prime}}^{*}\left(\theta^{\prime}, \phi^{\prime}\right) Y_{l m_{l}}(\theta, \phi) \\
& \quad \times\left\langle s m_{j}-m_{l}^{\prime}\left|V^{t m_{t}}\left(\boldsymbol{p}^{\prime}, \boldsymbol{p}\right)\right| s m_{j}-m_{l}\right\rangle
\end{aligned}
$$

needs to be evaluated. Here $c\left(l, s, j ; m_{l}, m_{j}-m_{l}, m_{j}\right)$ are the standard Clebsch-Gordan coefficients, and $Y_{l m_{l}}(\theta, \phi)$ the spherical harmonics calculated for the angles corresponding to the directions of the momenta $\boldsymbol{p}^{\prime}$ and $\boldsymbol{p}$. This quantity does not actually depend on $m_{j}$, so instead of Eq. (5) we can calculate

$$
H\left(l^{\prime}, l, s, j\right) \equiv \frac{1}{2 j+1} \sum_{m_{j}=-j}^{j}\left\langle p^{\prime}\left(l^{\prime} s\right) j m_{j}|V| p(l s) j m_{j}\right\rangle .
$$

Since now the integrand is a scalar, it is possible to reduce the number of integrals to one:

$$
\begin{aligned}
& H\left(l^{\prime}, l, s, j\right)=8 \pi^{2} \int_{-1}^{1} d\left(\cos \theta^{\prime}\right) \frac{1}{2 j+1} \sum_{m_{j}=-j}^{j} \\
& \times \sum_{m_{l}^{\prime}=-l^{\prime}}^{l^{\prime}} c\left(l^{\prime}, s, j ; m_{l}^{\prime}, m_{j}-m_{l}^{\prime}, m_{j}\right) \\
& \times \sum_{m_{l}=-l}^{l} c\left(l, s, j ; m_{l}, m_{j}-m_{l}, m_{j}\right) \\
& \times Y_{l^{\prime} m_{l}^{\prime}}\left(\theta^{\prime}, 0\right) Y_{l m_{l}}^{*}(0,0) \\
& \times\left\langle s m_{j}-m_{l}^{\prime}\left|V\left(\boldsymbol{p}^{\prime}, \boldsymbol{p}\right)\right| s m_{j}-m_{l}\right\rangle .
\end{aligned}
$$

In the NN system we can choose the z-axis to be

$$
\boldsymbol{p}=(0,0, p)
$$

Then the direction of the vector $\boldsymbol{p}^{\prime}$ is given as

$$
\boldsymbol{p}^{\prime}=\left(p^{\prime} \sin \theta^{\prime}, 0, p^{\prime} \cos \theta^{\prime}\right) \text {. }
$$

Most importantly however, the matrix element in the $2 \mathrm{~N}$ spin space,

$$
\begin{aligned}
& \left\langle s m_{j}-m_{l}^{\prime}\left|V\left(\boldsymbol{p}^{\prime}, \boldsymbol{p}\right)\right| s m_{j}-m_{l}\right\rangle= \\
& \left\langle s m_{j}-m_{l}^{\prime}\right| \sum_{i=1}^{6} f_{i}\left(p^{\prime}, p, x\right) \\
& \quad w_{i}\left(\boldsymbol{\sigma}(1), \boldsymbol{\sigma}(2), \boldsymbol{p}^{\prime}, \boldsymbol{p}\right)\left|s m_{j}-m_{l}\right\rangle
\end{aligned}
$$

can be calculated analytically 1 . The three sums in Eq. (7) over $m_{j}, m_{l}^{\prime}$ and $m_{l}$ can be written out explicitly so the integrand over $x \equiv \cos \theta^{\prime}$ can be prepared once for all NN potentials that are represented in the form of Eq. (3). The resulting expression is given in terms of $p, p^{\prime}, x$ and the expansion coefficients $f(i) \equiv f_{i}\left(p^{\prime}, p, x\right)$. As example, evaluating Eq. (7) for the ${ }^{1} S_{0}$ channel leads to

$$
\begin{aligned}
H(0,0,0,0)= & 2 \pi \int_{-1}^{1} d x\left(f(1)-3 f(2)+f(4) p^{2} p^{2}\left(x^{2}-1\right)\right. \\
& -f(5)\left(p^{2}+p^{\prime 2}+2 p p^{\prime} x\right) \\
& \left.-f(6)\left(p^{2}+p^{\prime 2}-2 p p^{\prime} x\right)\right)
\end{aligned}
$$

Further examples are collected in Appendix $\mathrm{A}$.

1 For this calculation symbolic software like Mathematica (C) 11] proves very useful. Here it is particularly simple with the concept of the Kronecker product which makes such matrix elements simple matrix elements in the four-dimensional space. 


\section{Application of the new method to the $2 \mathrm{~N}$ potential}

In this section we want to give two examples a PWD of an NN potential based on our new method and compare with results obtained in the traditional way. First we consider the one-boson exchange potential BonnB [8]. Here we use the operator form of this potential presented in 6 . In Figs. 1 6 we show matrix elements $\left\langle p^{\prime}\left(l^{\prime} s\right) j m_{j}\left|V^{t m_{t}}\right|\right.$ $\left.p_{0}(l s) j m_{j}\right\rangle$ for several partial waves for a fixed value of $p_{0}=1 \mathrm{fm}^{-1}$ as a function of the momentum $p^{\prime}$. In Figs. 1 through 3 selected partial wave projected potential amplitudes for the BonnB potential are shown. The agreement of the matrix elements calculated with our operator based formulation and the original PWD based on a helicity formulation [9] is excellent. That is not too surprising, in both cases the numerical accuracy is determined by the integration over $\cos \theta^{\prime}$.

As second example we take the neutron-proton version of the chiral NNLO potential [7] and use the same set of the parameters as given in [6]. In Figs. 46 6] selected partial wave projected potential amplitudes are shown for this potential. Again, the agreement between the two methods is excellent.

\section{Extension to Three-Nucleon Forces}

The arguments used in Sec. 2 for the $2 \mathrm{~N}$ potential can be applied to any three-nucleon $(3 \mathrm{~N})$ force. Now we use the $3 \mathrm{~N}$ states $|p q \beta\rangle[12$ in the so-called $L S$-coupling

$$
|p q \beta\rangle \equiv\left|p q(l \lambda) L\left(s \frac{1}{2}\right) S(L S) J M_{J}\right\rangle\left|\left(t \frac{1}{2}\right) T m_{T}\right\rangle,
$$

where the quantum numbers for the relative angular momenta $l$ (within the pair (23)) and $\lambda$ (between the pair (23) and nucleon 1) are coupled to the total angular momentum $L$. In the spin space the spin of the (23) pair is coupled with the spin $\frac{1}{2}$ of nucleon 1 to the total spin $S$. Finally $L$ and $S$ are coupled to the total $3 \mathrm{~N}$ angular momentum $J$ with the projection $M_{J}$. The isospin $3 \mathrm{~N}$ state, where we couple the total isospin of the (23) subsystem $t$ with the isospin $\frac{1}{2}$ of the third nucleon to the total $3 \mathrm{~N}$ isospin $T$ with the projection $m_{T}$, is constructed in the same way as the $3 \mathrm{~N}$ spin state. Since we want the states $|p q \beta\rangle$ to be antisymmetric with respect to the exchange of particles 2 and 3 , we require additionally that $(-1)^{l+s+t}=-1$.

We start with the eight-dimensional integral

$$
\begin{aligned}
& \left\langle p^{\prime} q^{\prime}\left(l^{\prime} \lambda^{\prime}\right) L^{\prime}\left(s^{\prime} \frac{1}{2}\right) S^{\prime}\left(L^{\prime} S^{\prime}\right) J M_{J}\right| \\
& V^{3 N}\left|p q(l \lambda) L\left(s \frac{1}{2}\right) S(L S) J M_{J}\right\rangle \\
& =\int d \hat{p}^{\prime} \int d \hat{q}^{\prime} \int d \hat{p} \int d \hat{q} \\
& \sum_{m_{L^{\prime}}} c\left(L^{\prime}, S^{\prime}, J ; m_{L^{\prime}}, M_{J}-m_{L^{\prime}}, M_{J}\right) \\
& \sum_{m_{L}} c\left(L, S, J ; m_{L}, M_{J}-m_{L}, M_{J}\right) \\
& \mathcal{Y}_{l^{\prime}, \lambda^{\prime}}^{*}, m_{L^{\prime}}\left(\hat{p}^{\prime}, \hat{q}^{\prime}\right) \mathcal{Y}_{l, \lambda}^{L, m_{L}}(\hat{p}, \hat{q}) \\
& \left\langle\left(s \frac{1}{2}\right) S^{\prime} M_{J}-m_{L^{\prime}}\left|V^{3 N}\left(\boldsymbol{p}^{\prime}, \boldsymbol{q}^{\prime}, \boldsymbol{p}, \boldsymbol{q}\right)\right|\left(s \frac{1}{2}\right) S M_{J}-m_{L}\right\rangle,
\end{aligned}
$$

where

$$
\begin{aligned}
\mathcal{Y}_{l, \lambda}^{L, m_{L}}(\hat{p}, \hat{q}) \equiv & \sum_{\substack{m_{l}=-l\\
}}^{l} c\left(l, \lambda, L ; m_{l}, m_{L}-m_{l}, m_{L}\right) \\
& \times Y_{l, m_{l}}(\hat{p}) Y_{\lambda, m_{L}-m_{l}}(\hat{q}) .
\end{aligned}
$$

This quantity is independent of $M_{J}$, and instead of Eq. (13) we can calculate

$$
\begin{aligned}
& G\left(l^{\prime}, \lambda^{\prime}, L^{\prime}, s^{\prime}, S^{\prime}, l, \lambda, L, s, S, J\right) \\
& \equiv \frac{1}{2 J+1} \sum_{M_{J}=-J}^{J}\left\langle p^{\prime} q^{\prime}\left(l^{\prime} \lambda^{\prime}\right) L^{\prime}\left(s^{\prime} \frac{1}{2}\right) S^{\prime}\left(L^{\prime} S^{\prime}\right) J M_{J}\right| \\
& V^{3 N}\left|p q(l \lambda) L\left(s \frac{1}{2}\right) S(L S) J M_{J}\right\rangle .
\end{aligned}
$$

Since the integrand is now a scalar, it is possible to reduce the number of integrals from eight to five. Namely we take first $\hat{p}=\hat{z}$ and then consider all scalar products among the $\hat{p}, \hat{q}, \hat{p}^{\prime}$ and $\hat{q}^{\prime}$ unit vectors. They depend on the following quantities: $\theta_{q}, \theta_{p^{\prime}}, \theta_{q^{\prime}}, \phi_{p^{\prime}}-\phi_{q}, \phi_{q^{\prime}}-\phi_{q}$ and $\phi_{p^{\prime}}-\phi_{q^{\prime}}$. Since the last three are not independent, it is possible to set additionally $\phi_{q}=0$. Of course, taking $\hat{p}=\hat{z}$ and $\phi_{q}=0$ is only one possibility. The best choice (from the computational point of view) of the five integration variables might depend on the form of $V^{3 N}\left(\boldsymbol{p}^{\prime}, \boldsymbol{q}^{\prime}, \boldsymbol{p}, \boldsymbol{q}\right)$, which is an operator in the $3 \mathrm{~N}$ spin space. This quantity $G$ has to be multiplied by the matrix element of isospin operator $\hat{I}$

$$
\left\langle\left(t^{\prime} \frac{1}{2}\right) T^{\prime} m_{T^{\prime}}|\hat{I}|\left(t \frac{1}{2}\right) T m_{T}\right\rangle,
$$

which can be worked out exactly, independent from the momentum and spin spaces.

\section{Numerical example for a $3 \mathrm{~N}$ force}

We consider just one example of the $3 \mathrm{~N}$ force: a two-pionexchange contribution to the chiral NNLO $3 \mathrm{~N}$ force as given in 13], $V_{T P E}^{3 N F}$. In particular we take the part of $V_{T P E}^{3 N F}$ which is symmetric under the exchange of nucleons 2 and 3 

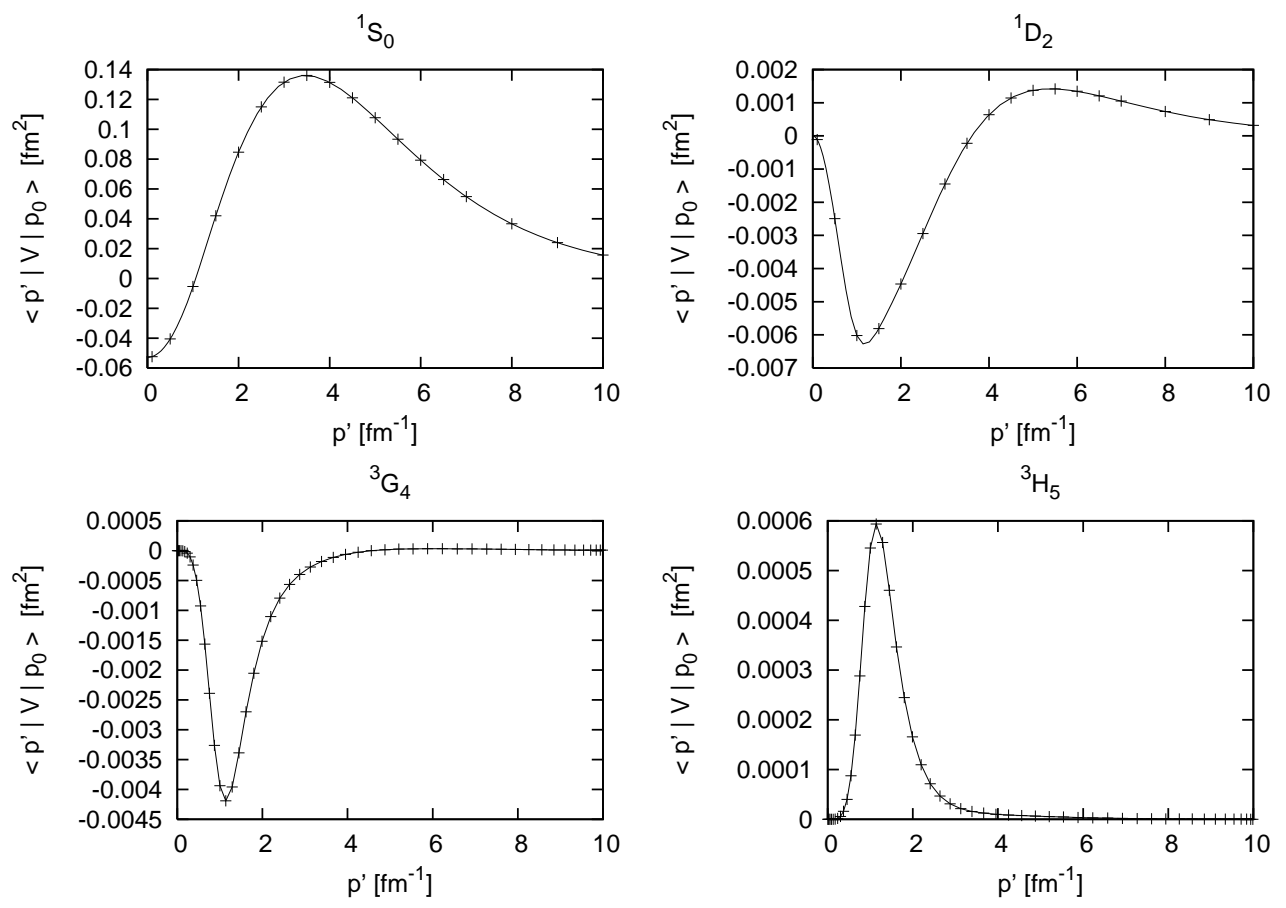

Fig. 1. Comparison of the traditional (crosses) and the new (solid line) method of PWD for the Bonn B potential. The matrix elements of selected uncoupled channels with $t=1\left({ }^{1} S_{0},{ }^{1} D_{2}\right)$ and $t=0\left({ }^{3} G_{4},{ }^{3} H_{5}\right)$ are shown for a fixed value of $p_{0}=1 \mathrm{fm}{ }^{-1}$ as a function of the $p^{\prime}$ momentum.

$$
\begin{aligned}
V^{(1)}= & F_{1} \boldsymbol{\sigma}_{2} \cdot \boldsymbol{q}_{2} \boldsymbol{\sigma}_{3} \cdot \boldsymbol{q}_{3} \boldsymbol{\tau}_{2} \cdot \boldsymbol{\tau}_{3} \\
& +F_{2} \boldsymbol{\sigma}_{1} \cdot\left(\boldsymbol{q}_{2} \times \boldsymbol{q}_{3}\right) \boldsymbol{\sigma}_{2} \cdot \boldsymbol{q}_{2} \boldsymbol{\sigma}_{3} \cdot \boldsymbol{q}_{3} \boldsymbol{\tau}_{1} \cdot\left(\boldsymbol{\tau}_{2} \times \boldsymbol{\tau}_{3}\right)
\end{aligned}
$$

where $\boldsymbol{q}_{i} \equiv \boldsymbol{p}_{i}{ }_{i}-\boldsymbol{p}_{i}$ and $\boldsymbol{p}_{i}\left(\boldsymbol{p}^{\prime}{ }_{i}\right)$ is the initial (final) momentum of nucleon $i$. Further, $g_{A}, m_{\pi}$ and $F_{\pi}$ refer to the nucleon axial vector coupling constant, pion mass and decay constants, respectively, while $c_{i}$ are low-energy constants from the subleading pion-nucleon Lagrangian. The scalar functions $F_{1}$ and $F_{2}$ are

$$
\begin{aligned}
F_{1}= & \left(\frac{g_{A}}{2 F_{\pi}}\right)^{2} \frac{1}{\left(\boldsymbol{q}_{2}^{2}+m_{\pi}^{2}\right)\left(\boldsymbol{q}_{3}^{2}+m_{\pi}^{2}\right)} \\
& \times\left(-\frac{4 c_{1} m_{\pi}^{2}}{F_{\pi}^{2}}+\frac{2 c_{3}}{F_{\pi}^{2}} \boldsymbol{q}_{2} \cdot \boldsymbol{q}_{3}\right)
\end{aligned}
$$

and

$$
F_{2}=\left(\frac{g_{A}}{2 F_{\pi}}\right)^{2} \frac{1}{\left(\boldsymbol{q}_{2}^{2}+m_{\pi}^{2}\right)\left(\boldsymbol{q}_{3}^{2}+m_{\pi}^{2}\right)} \frac{c_{4}}{F_{\pi}^{2}}
$$

We consider first the two isospin matrix elements

$$
\begin{aligned}
& \hat{I}_{1}\left(t^{\prime}, T^{\prime}, m_{T^{\prime}}, t, T, m_{T}\right) \\
& \quad \equiv\left\langle\left(t^{\prime} \frac{1}{2}\right) T^{\prime} m_{T^{\prime}}\left|\boldsymbol{\tau}_{2} \cdot \boldsymbol{\tau}_{3}\right|\left(t \frac{1}{2}\right) T m_{T}\right\rangle
\end{aligned}
$$

and

$$
\begin{aligned}
& \hat{I}_{2}\left(t^{\prime}, T^{\prime}, m_{T^{\prime}}, t, T, m_{T}\right) \\
& \quad \equiv\left\langle\left(t^{\prime} \frac{1}{2}\right) T^{\prime} m_{T^{\prime}}\left|\boldsymbol{\tau}_{1} \cdot\left(\boldsymbol{\tau}_{2} \times \boldsymbol{\tau}_{3}\right)\right|\left(t \frac{1}{2}\right) T m_{T}\right\rangle .
\end{aligned}
$$

The matrix elements $\hat{I}_{1}\left(t^{\prime}, T^{\prime}, m_{T^{\prime}}, t, T, m_{T}\right)$ are particularly simple and are given as

$$
\begin{aligned}
& \hat{I}_{1}\left(t^{\prime}, T^{\prime}, m_{T^{\prime}}, t, T, m_{T}\right) \\
& \quad=(2 t(t+1)-3) \delta_{t, t^{\prime}} \delta_{T, T^{\prime}} \delta_{m_{T}, m_{T^{\prime}}}
\end{aligned}
$$

The (purely imaginary) matrix elements

$\hat{I}_{2}\left(t^{\prime}, T^{\prime}, m_{T^{\prime}}, t, T, m_{T}\right)$ can be written as

$$
\begin{aligned}
& \hat{I}_{2}\left(t^{\prime}, T^{\prime}, m_{T^{\prime}}, t, T, m_{T}\right) \\
& \quad=i \sqrt{3}(-1)^{t+1} \delta_{t+t^{\prime}, 1} \delta_{T, \frac{1}{2}} \delta_{T^{\prime}, \frac{1}{2}} \delta_{m_{T}, m_{T^{\prime}}} .
\end{aligned}
$$

From Eqs. (22) and (23) we defer immediately that the two parts of the considered $3 \mathrm{~N}$ force (17) will not contribute simultaneously to the same matrix element $G\left(l^{\prime}, \lambda^{\prime}, L^{\prime}, s^{\prime}, S^{\prime}, l, \lambda, L, s, S, J\right)$.

For this first simple study we construct $16|\beta\rangle$ states for $J=\frac{1}{2}$ and positive parity $\pi=(-1)^{l+\lambda}$ satisfying the additional condition $l \leq 2$ and $\lambda \leq 2$. Their quantum numbers are given in Table 1

Next we perform the steps described in Sec. 4 and obtain 256 integrands $\tilde{G}\left(l^{\prime}, \lambda^{\prime}, L^{\prime}, s^{\prime}, S^{\prime}, l, \lambda, L, s, S, J\right) \equiv$ $\tilde{G}\left(\beta^{\prime}, \beta\right)$ such that

$$
\begin{aligned}
G\left(\beta^{\prime}, \beta\right) & \equiv\left\langle\left(t^{\prime} \frac{1}{2}\right) T^{\prime} m_{T^{\prime}}\left|\left\langle p^{\prime} q^{\prime} \beta^{\prime}\left|V^{3 N}\right| p q \beta\right\rangle\right|\left(t \frac{1}{2}\right) T m_{T}\right\rangle \\
& \equiv \int d \hat{p}^{\prime} \int d \hat{q}^{\prime} \int d \hat{p} \int d \hat{q} \tilde{G}\left(\beta^{\prime}, \beta\right) .
\end{aligned}
$$

Here we show just few (relatively simple) examples. (Note that in the first two cases the scalar nature of the 
Table 1. List of $|\beta\rangle$ states for $J^{\pi}=\frac{1}{2}^{+}, l \leq 2$ and $\lambda \leq 2$.

\begin{tabular}{ccccccc}
\hline$\beta$ & $\mathrm{l}$ & $\mathrm{s}$ & $\lambda$ & $\mathrm{L}$ & $\mathrm{S}$ & $\mathrm{t}$ \\
\hline 1 & 0 & 0 & 0 & 0 & $\frac{1}{2}$ & 1 \\
2 & 0 & 1 & 0 & 0 & $\frac{1}{2}$ & 0 \\
3 & 0 & 1 & 2 & 2 & $\frac{3}{2}$ & 0 \\
4 & 1 & 0 & 1 & 0 & $\frac{1}{2}$ & 0 \\
5 & 1 & 0 & 1 & 1 & $\frac{1}{2}$ & 0 \\
6 & 1 & 1 & 1 & 0 & $\frac{1}{2}$ & 1 \\
7 & 1 & 1 & 1 & 1 & $\frac{1}{2}$ & 1 \\
8 & 1 & 1 & 1 & 1 & $\frac{3}{2}$ & 1 \\
9 & 1 & 1 & 1 & 2 & $\frac{3}{2}$ & 1 \\
10 & 2 & 1 & 0 & 2 & $\frac{3}{2}$ & 0 \\
11 & 2 & 0 & 2 & 0 & $\frac{1}{2}$ & 1 \\
12 & 2 & 0 & 2 & 1 & $\frac{1}{2}$ & 1 \\
13 & 2 & 1 & 2 & 0 & $\frac{1}{2}$ & 0 \\
14 & 2 & 1 & 2 & 1 & $\frac{1}{2}$ & 0 \\
15 & 2 & 1 & 2 & 1 & $\frac{3}{2}$ & 0 \\
16 & 2 & 1 & 2 & 2 & $\frac{3}{2}$ & 0
\end{tabular}

$\tilde{G}\left(\beta^{\prime}, \beta\right)$ functions is clearly visible. $)$

$$
\begin{aligned}
\tilde{G}(1,1)= & -\frac{1}{16 \pi^{2}} F_{1} \hat{I}_{1}\left(1, T^{\prime}, m_{T^{\prime}}, 1, T, m_{T}\right) \boldsymbol{q}_{2} \cdot \boldsymbol{q}_{3}, \\
\tilde{G}(2,1)= & -\frac{i}{16 \pi^{2} \sqrt{3}} F_{2} \hat{I}_{2}\left(0, T^{\prime}, m_{T^{\prime}}, 1, T, m_{T}\right) \\
& \times\left(\left(\boldsymbol{q}_{2} \cdot \boldsymbol{q}_{3}\right)^{2}-\boldsymbol{q}_{2}{ }^{2} \boldsymbol{q}_{3}{ }^{2}\right), \\
\tilde{G}(5,11)= & \frac{1}{2 \sqrt{3}} F_{2} \hat{I}_{2}\left(0, T^{\prime}, m_{T^{\prime}}, 1, T, m_{T}\right)\left(\boldsymbol{q}_{2} \cdot \boldsymbol{q}_{3}\right) \\
& \times \mathcal{Y}_{2,2}^{0,0}(\hat{p}, \hat{q})\left(\sqrt{2}\left(q_{4 x}-i q_{4 y}\right) \mathcal{Y}_{1,1}^{* 1,-1}\left(\hat{p}^{\prime}, \hat{q}^{\prime}\right)\right. \\
& +2 q_{4 z} \mathcal{Y}_{1,1}^{* 1,0}\left(\hat{p}^{\prime}, \hat{q}^{\prime}\right) \\
& \left.-\sqrt{2}\left(q_{4 x}+i q_{4 y}\right) \mathcal{Y}_{1,1}^{* 1,1}\left(\hat{p}^{\prime}, \hat{q}^{\prime}\right)\right), \\
\tilde{G}(6,12)= & \frac{1}{6} F_{1} \hat{I}_{1}\left(1, T^{\prime}, m_{T^{\prime}}, 1, T, m_{T}\right) \mathcal{Y}_{1,1}^{* 0,0}\left(\hat{p}^{\prime}, \hat{q}^{\prime}\right) \\
& \left(\sqrt { 2 } \left(q_{2 z}\left(q_{3 x}+i q_{3 y}\right)\right.\right. \\
& \left.-\left(q_{2 x}+i q_{2 y}\right) q_{3 z}\right) \mathcal{Y}_{2,2}^{1,-1}(\hat{p}, \hat{q}) \\
& +2 i\left(q_{2 y} q_{3 x}-q_{2 x} q_{3 y}\right) \mathcal{Y}_{2,2}^{1,0}(\hat{p}, \hat{q}) \\
& +\sqrt{2}\left(q_{2 z}\left(q_{3 x}-i q_{3 y}\right)\right. \\
& \left.\left.-\left(q_{2 x}-i q_{2 y}\right) q_{3 z}\right) \mathcal{Y}_{2,2}^{1,1}(\hat{p}, \hat{q})\right),
\end{aligned}
$$

\begin{tabular}{|c|c|c|c|c|}
\hline$N$ & $G(1,1)$ & $G(2,1)$ & $G(6,12)$ & $G(5,11)$ \\
\hline 12 & 443.565 & 1200.160 & -5.52616 & -5.24720 \\
\hline 24 & 443.618 & 1200.223 & -5.49311 & -5.48527 \\
\hline 36 & 443.618 & 1200.219 & -5.49290 & -5.48630 \\
\hline 48 & 443.618 & 1200.219 & -5.49290 & -5.48626 \\
\hline $\begin{array}{l}\text { standar } \\
\text { PWD }\end{array}$ & 443.618 & 1200.219 & .49274 & 7 \\
\hline
\end{tabular}

where $\boldsymbol{q}_{4} \equiv \boldsymbol{q}_{2} \times \boldsymbol{q}_{3}$ and the Cartesian components of the $\boldsymbol{q}_{i}$ vectors are denoted as $q_{i x}, q_{i y}$ and $q_{i z}$. Of course, vectors $\boldsymbol{q}_{i}$ are now expressed in terms of the initial and final Jacobi momenta in the following way

$$
\begin{aligned}
& \boldsymbol{q}_{1}=\boldsymbol{q}^{\prime}-\boldsymbol{q} \\
& \boldsymbol{q}_{2}=\boldsymbol{p}^{\prime}-\frac{1}{2} \boldsymbol{q}^{\prime}-\left(\boldsymbol{p}-\frac{1}{2} \boldsymbol{q}\right) \\
& \boldsymbol{q}_{3}=-\boldsymbol{p}^{\prime}-\frac{1}{2} \boldsymbol{q}^{\prime}-\left(-\boldsymbol{p}-\frac{1}{2} \boldsymbol{q}\right) .
\end{aligned}
$$

Many of the $\tilde{G}\left(\beta^{\prime}, \beta\right)$ functions are very lengthy. Using symbolic manipulations software like Mathematica (c) [11]
Table 2. Stability of the selected five fold integrals with respect to the number of Gaussian points $N$ (see text). In the last row the values obtained with standard PWD 14,15] are given. All matrix elements are in $\mathrm{fm}^{5}$.

Table 3. Stability of the selected six fold integrals with respect to the number of Gaussian points $N$ (see text). All matrix elements are in $\mathrm{fm}^{5}$.

\begin{tabular}{crrrr}
\hline$N$ & $G(1,1)$ & $G(2,1)$ & $G(6,12)$ & $G(5,11)$ \\
\hline 12 & 443.510 & 1200.365 & -5.55695 & -5.14678 \\
24 & 443.619 & 1200.218 & -5.49277 & -5.48785 \\
36 & 443.618 & 1200.219 & -5.49290 & -5.48626 \\
48 & 443.618 & 1200.219 & -5.49290 & -5.48626
\end{tabular}

one can write down these expressions directly as a part of a Fortran or a $\mathrm{C}$ code. The expressions given above have to be integrated over five angles. With the choice of variables discussed in Sec. 4. we calculated the five fold integrals for fixed magnitudes of the momenta $p, q, p^{\prime}$ and $q^{\prime}$. They were chosen quite arbitrarily: $p=1 \mathrm{fm}^{-1}, q=2 \mathrm{fm}^{-1}, p^{\prime}=$ $3 \mathrm{fm}^{-1}, q^{\prime}=4 \mathrm{fm}^{-1}$. For the isospin quantum numbers we assumed that $T=T^{\prime}=m_{T}=m_{T^{\prime}}=\frac{1}{2}$. The $3 \mathrm{~N}$ force parameters were taken as $g_{A}=1.29, F_{\pi}=92.4 \mathrm{MeV}, m_{\pi}=$ $138.0 \mathrm{MeV} / \mathrm{c}^{2}, c_{1}=-0.81 \mathrm{GeV}^{-1}, c_{3}=-3.4 \mathrm{GeV}^{-1}, c_{4}=$ $3.4 \mathrm{GeV}^{-1}$. Such single five fold integrals can be easily calculated on a PC. For a simple test we chose the same number of Gaussian integral points $N$ in each of the five dimensions and checked the stability of the integrals with respect to $N$. Even in this most simple way, we found a good convergence, which is shown in Table 2. To check numerically the scalar nature of our integrands we calculated also the corresponding six fold integrals, where $\phi_{q}$ was an additional integration variable and checked their stability (see Table 3). We see from Tables 2 and 3 that the results from the five and six fold integrations agree very well with each other. We calculated the same matrix elements using standard PWD 14,15. The results are shown in the last row of Table 2. They agree very well with the numbers obtained using the new method. Thus we conclude that the presented procedure might be used to efficiently automate the very cumbersome standard PWD of the $3 \mathrm{~N}$ force. Calculation of all matrix elements for the whole grids of $p, q, p^{\prime}$ and $q^{\prime}$ points, especially for bigger values of $J$, will require an implementation of the algorithm on parallel architectures. Implementing such a code is much simpler than implementing a code based on the traditional PWD. 


\section{Summary and Outlook}

We propose a new general method of calculating the PWD of any NN potential which is given in momentum space in terms of operators acting in the $2 \mathrm{~N}$ spin and isospin spaces. Once the expansion coefficients in the operator basis are determined, the matrix elements of interest are obtained as simple one dimensional integrals, independent from the particular form of the NN potential. We demonstrate this method with two examples, the one-bosonexchange BonnB potential and a recently derived chiral NNLO potential. In both cases the agreement between our new method and traditionally employed one to obtaining partial wave projected matrix elements is perfect.

This method can be extended to treat three-nucleon forces, for which a traditional PWD is a formidable task. In order to show the power of our new method, we performed a simple feasibility study for the TPE chiral $3 \mathrm{~N}$ force. A very good agreement between the new method and standard PWD shows that the very complex traditional approach can be replaced by this straightforward and efficient algebraic method. An 'automated' PWD for the $3 \mathrm{NF}$ is of great importance in view of awaiting applications in the $3 \mathrm{~N}$ systems. The numerous contributions to the chiral N3LO $3 \mathrm{~N}$ force [16,17] can be handled only with such an efficient and simple procedure.

\section{Acknowledgments}

This work was supported by the Polish 2008-2011 science funds as the research project No. N N202 077435 and in part under the auspices of the U. S. Department of Energy, Office of Nuclear Physics under contract No. DE-FG02-93ER40756 with Ohio University. It was also partially supported by the Helmholtz Association through funds provided to the virtual institute "Spin and strong QCD"(VH-VI-231) and to the young investigator group "Few-Nucleon Systems in Chiral Effective Field Theory" (grant VH-NG-222) and by the European CommunityResearch Infrastructure Integrating Activity "Study of Strongly Interacting Matter" (acronym HadronPhysics2, Grant Agreement n. 227431) under the Seventh Framework Programme of EU. The numerical calculations have been partly performed on the supercomputer cluster of the JSC, Jülich, Germany.

\section{A Integrals for the $2 \mathrm{~N}$ potential}

This appendix collects the expressions for the partial wave decomposition of the NN potential for the partial waves with total $2 \mathrm{~N}$ angular momentum $j \leq 2$. The expressions for higher $j$ can be obtained on request from one of the authors (JG) either in analytical form or as Fortran or C code.

The second function for $j=0$ is $(H(0,0,0,0)$ was given in Sec. 2):

$$
\begin{aligned}
& H(1,1,1,0)=2 \pi \int_{-1}^{1} d x\{[f(1)+f(2)] x \\
& +2 f(3) p p^{\prime}\left(x^{2}-1\right)+f(4) p^{2} p^{\prime 2} x\left(1-x^{2}\right) \\
& \quad-f(5)\left[2 p^{\prime} p+x\left(p^{2}+p^{2}\right)\right] \\
& \left.+f(6)\left[2 p^{\prime} p-x\left(p^{2}+p^{2}\right)\right]\right\} .
\end{aligned}
$$

The functions for $j=1$ are given by

$$
\begin{aligned}
& H(1,1,0,1)=2 \pi \int_{-1}^{1} d x\{[f(1)+3 f(2)] x \\
& +f(4) p^{2} p^{\prime 2} x\left(x^{2}-1\right) \\
& -f(5) x\left[2 p^{\prime} p x+\left(p^{2}+p^{2}\right)\right] \\
& \left.+f(6) x\left[2 p^{\prime} p x-\left(p^{2}+p^{2}\right)\right]\right\} \text {, } \\
& H(1,1,1,1)=2 \pi \int_{-1}^{1} d x\{[f(1)+f(2)] x \\
& +f(3) p^{\prime} p\left(x^{2}-1\right) \\
& +f(5)\left[x\left(p^{2}+p^{2}\right)+p^{\prime} p\left(x^{2}+1\right)\right] \\
& \left.+f(6)\left[x\left(p^{2}+p^{2}\right)-p^{\prime} p\left(x^{2}+1\right)\right]\right\} \text {, } \\
& H(0,0,1,1)=\frac{2 \pi}{3} \int_{-1}^{1} d x\{3[f(1)+f(2)] \\
& +f(4) p^{2} p^{\prime 2}\left(1-x^{2}\right) \\
& +f(5)\left[p^{\prime 2}+p^{2}+2 p^{\prime} p x\right] \\
& \left.+f(6)\left[p^{\prime 2}+p^{2}-2 p^{\prime} p x\right]\right\} \text {, } \\
& H(0,2,1,1)=2 \pi \frac{\sqrt{2}}{3} \int_{-1}^{1} d x\left\{f(4) p^{2} p^{2}\left(x^{2}-1\right)\right. \\
& +f(5)\left[\left(3 x^{2}-1\right) p^{\prime 2}+2 p^{2}+4 p^{\prime} p x\right] \\
& \left.+f(6)\left[\left(3 x^{2}-1\right) p^{\prime 2}+2 p^{2}-4 p^{\prime} p x\right]\right\} \\
& H(2,0,1,1)=2 \pi \frac{\sqrt{2}}{3} \int_{-1}^{1} d x\left\{f(4) p^{2} p^{2}\left(x^{2}-1\right)\right. \\
& +f(5)\left[2 p^{2}+\left(x^{2}-1\right) p^{2}+4 p^{\prime} p x\right] \\
& \left.+f(6)\left[2 p^{\prime 2}+\left(x^{2}-1\right) p^{2}-4 p^{\prime} p x\right]\right\}
\end{aligned}
$$




$$
\begin{aligned}
& H(2,2,1,1)=\frac{\pi}{3} \int_{-1}^{1} d x\left\{3[f(1)+f(2)]\left(3 x^{2}-1\right)\right. \\
& \quad+18 f(3) p^{\prime} p x\left(x^{2}-1\right) \\
& \quad+f(4) p^{\prime 2} p^{2}\left(14 x^{2}-5-9 x^{4}\right) \\
& \quad+f(5)\left[\left(1-3 x^{2}\right)\left(p^{\prime 2}+p^{2}\right)-4 p^{\prime} p x\right] \\
& \left.\quad+f(6)\left[\left(1-3 x^{2}\right)\left(p^{\prime 2}+p^{2}\right)+4 p^{\prime} p x\right]\right\}
\end{aligned}
$$

The functions for $j=2$ are given by

$$
\begin{aligned}
& H(2,2,0,2)=\pi \int_{-1}^{1} d x\left(1-3 x^{2}\right)\{[-f(1)+3 f(2)] \\
& -f(4) p^{\prime 2} p^{2}\left(x^{2}-1\right) \\
& +f(5)\left[\left(p^{\prime 2}+p^{2}\right)+2 p^{\prime} p x\right] \\
& \left.+f(6)\left[\left(p^{\prime 2}+p^{2}\right)-2 p^{\prime} p x\right]\right\}, \\
& H(2,2,1,2)=\pi \int_{-1}^{1} d x\left\{[f(1)+f(2)]\left(3 x^{2}-1\right)\right. \\
& +2 f(3) p^{\prime} p x\left(x^{2}-1\right) \\
& +f(4) p^{\prime 2} p^{2}\left[x^{4}-2 x^{2}+1\right] \\
& +f(5)\left[\left(3 x^{2}-1\right)\left(p^{\prime 2}+p^{2}\right)+4 p^{\prime} p x^{3}\right] \\
& \left.+f(6)\left[x\left(3 x^{2}-1\right)\left(p^{2}+p^{2}\right)-4 p^{\prime} p x^{3}\right]\right\} \text {, } \\
& H(1,1,1,2)=\frac{2 \pi}{5} \int_{-1}^{1} d x\{5 x[f(1)+f(2)] \\
& +5 f(3) p^{\prime} p\left(1-x^{2}\right) \\
& +2 f(4) p^{\prime 2} p^{2} x\left(1-x^{2}\right) \\
& +f(5)\left[x\left(p^{2}+p^{2}\right)+p^{\prime} p\left(3 x^{2}-1\right)\right] \\
& \left.+f(6)\left[x\left(p^{\prime 2}+p^{2}\right)-p^{\prime} p\left(3 x^{2}-1\right)\right]\right\}, \\
& H(1,3,1,2)=2 \pi \frac{\sqrt{6}}{5} \int_{-1}^{1} d x\left\{f(4) p^{2} p^{2} x\left(x^{2}-1\right)\right. \\
& +f(5)\left[p^{\prime 2} x\left(5 x^{2}-3\right)+2 p^{2} x+2 p^{\prime} p\left(3 x^{2}-1\right)\right] \\
& \left.+f(6)\left[p^{\prime 2} x\left(5 x^{2}-3\right)+2 p^{2} x-2 p^{\prime} p\left(3 x^{2}-1\right)\right]\right\},
\end{aligned}
$$

$$
\begin{aligned}
& H(3,1,1,2)=2 \pi \frac{\sqrt{6}}{5} \int_{-1}^{1} d x\left\{f(4) p^{\prime 2} p^{2} x\left(x^{2}-1\right)\right. \\
& +f(5)\left[2 p^{\prime 2}+p^{2} x\left(5 x^{2}-3\right)+2 p^{\prime} p\left(3 x^{2}-1\right)\right] \\
& \left.\quad+f(6)\left[2 p^{\prime 2}+p^{2} x\left(5 x^{2}-3\right)-2 p^{\prime} p\left(3 x^{2}-1\right)\right]\right\}
\end{aligned}
$$

$$
\begin{aligned}
& H(3,3,1,2)=\frac{\pi}{5} \int_{-1}^{1} d x\left\{5 x\left(5 x^{2}-3\right)[f(1)+f(2)]\right. \\
& +10 f(3) p^{\prime} p\left(1+5 x^{4} 6 x^{2}\right) \\
& +f(4) p^{\prime 2} p^{2} x\left(44 x^{2}-25 x^{4}-19\right) \\
& \quad+f(5)\left[\left(p^{\prime 2}+p^{2}\right) x\left(3-5 x^{2}\right)+2 p^{\prime} p\left(1-3 x^{2}\right)\right] \\
& \left.\quad+f(6)\left[\left(p^{\prime 2}+p^{2}\right) x\left(3-5 x^{2}\right)-2 p^{\prime} p\left(1-3 x^{2}\right)\right]\right\} .
\end{aligned}
$$

\section{References}

1. Ch. Elster, W. Schadow, A. Nogga and W. Glöckle, Few Body Syst. 27, 83 (1999).

2. H. Liu, Ch. Elster and W. Glöckle, Phys. Rev. C 72, 054003 (2005).

3. I. Fachruddin, Ch. Elster, W. Glöckle, Phys. Rev. C63, 054003 (2001).

4. S. Bayegan, M. R. Hadizadeh and M. Harzchi, Phys. Rev. C77, 064005 (2008).

5. W. Glöckle, Ch. Elster, J. Golak, R. Skibiński, H. Witała, H. Kamada, arXiv:0906.0321 Few-Body Syst. DOI 10.1007/s00601-009-0064-1.

6. I. Fachruddin, Ch. Elster, W. Glöckle, J. Golak, D. Rozpędzik, R. Skibiński, K. Topolnicki, H. Witała, in preparation.

7. E. Epelbaum, H. W. Hammer and U. G. Meissner, arXiv:0811.1338 [nucl-th].

8. R. Machleidt, Adv. Nucl. Phys. 19, 189 (1989).

9. R. Machleidt, K. Holinde, Ch. Elster, Physics Reports 149, 1 (1987).

10. L. Wolfenstein, Phys. Rev. 96, 1654 (1954).

11. Wolfram Research, Inc., Mathematica (c), Version 7.0, Champaign, IL (2008).

12. W. Glöckle, The Quantum Mechanical Few-Body Problem, Springer-Verlag, Berlin-Heidelberg, (1983).

13. E. Epelbaum, A. Nogga, W. Glöckle, H. Kamada, Ulf-G. Meißner and H. Witała, Phys. Rev. C66, 064001 (2002).

14. S. A. Coon, W. Glöckle, Phys. Rev. C23, 1790 (1981).

15. D. Hüber, H. Witała, A. Nogga, W. Glöckle, and H. Kamada, Few-Body Syst. 22, 107 (1997).

16. V. Bernard, E. Epelbaum, H. Krebs and U. G. Meissner, Phys. Rev. C 77, 064004 (2008).

17. S. Ishikawa and M. R. Robilotta, Phys. Rev. C 76, 014006 (2007). 
J. Golak et al.: A new way to perform partial wave decompositions of few-nucleon forces
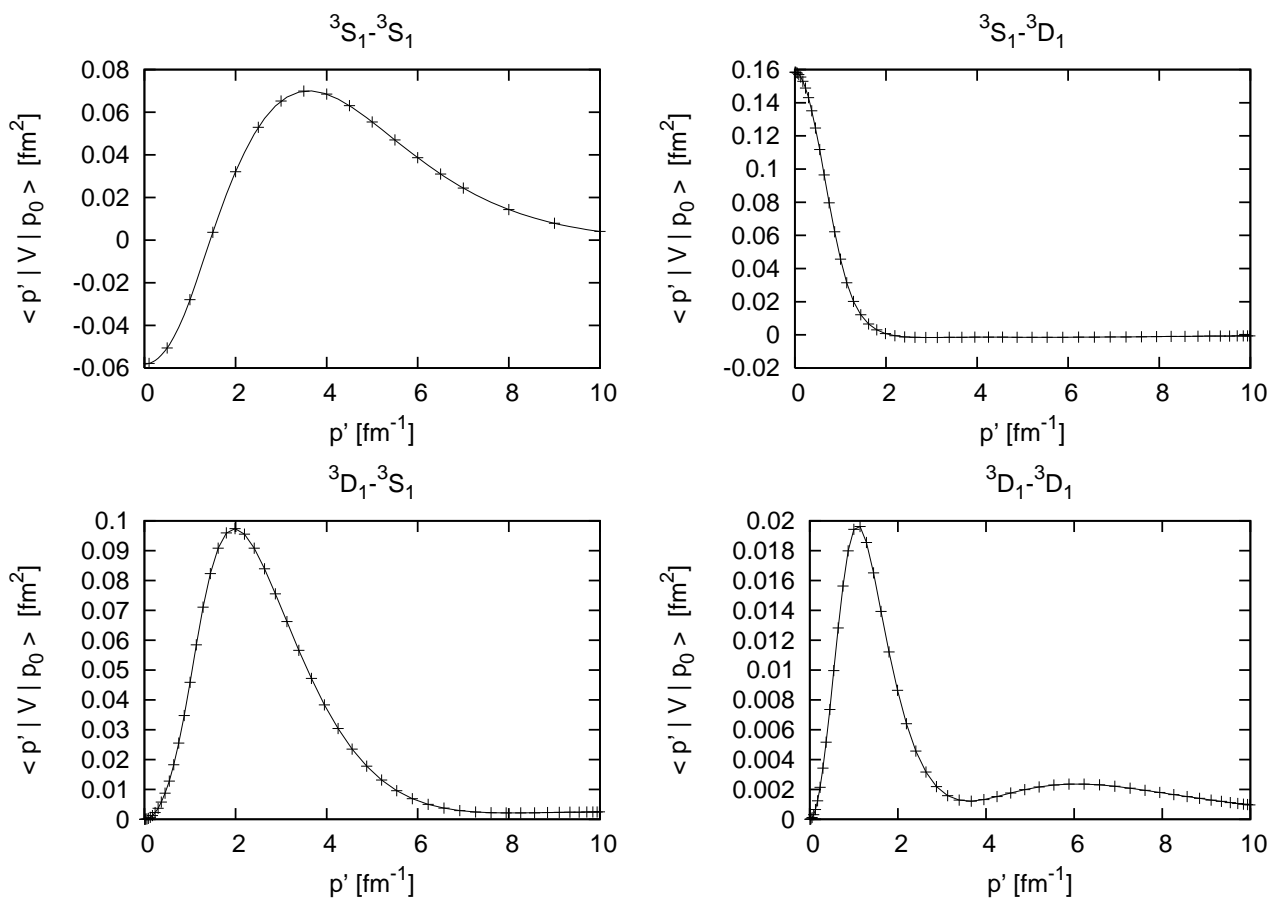

Fig. 2. The same as in Fig. 1 for one selected coupled channel case with $t=0$.
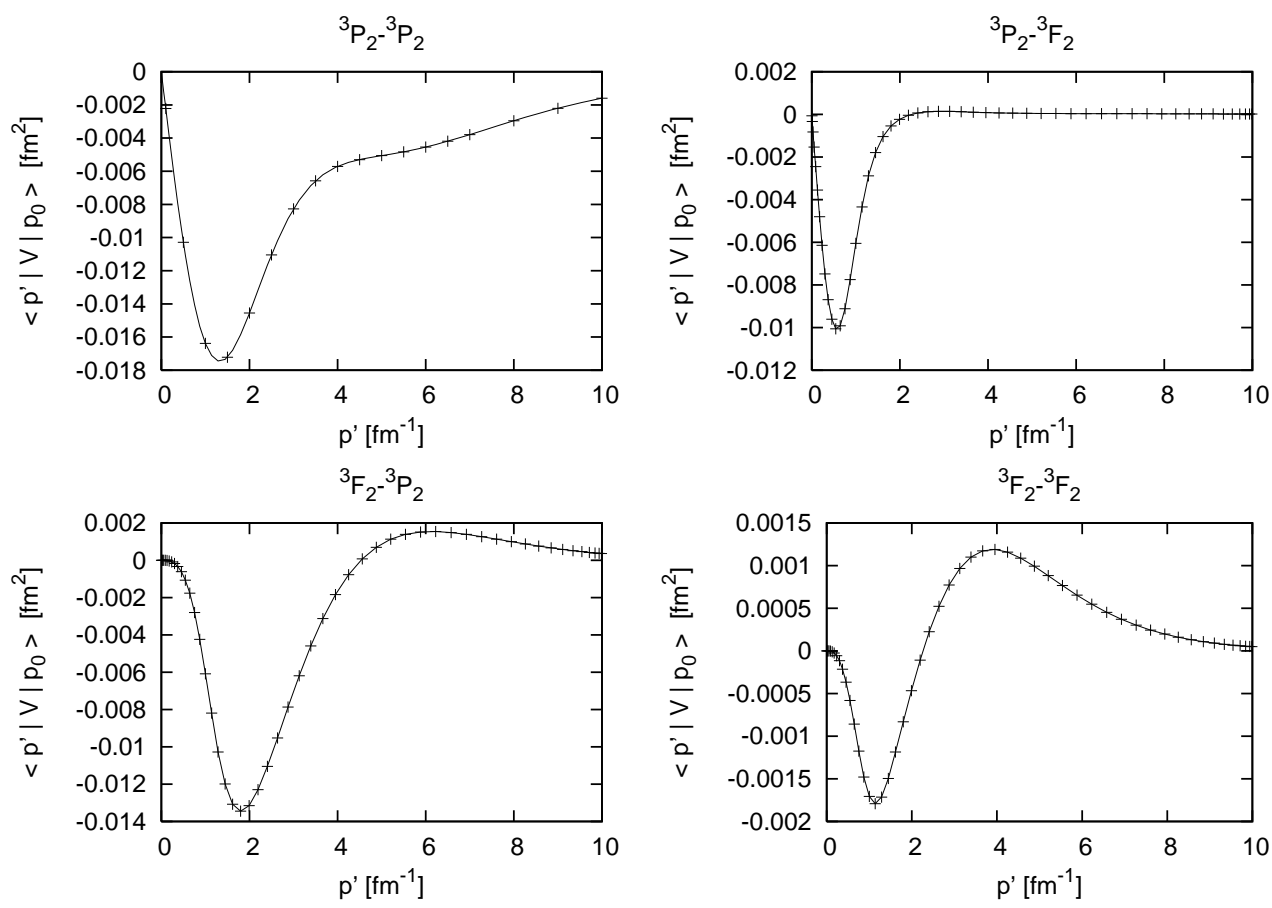

Fig. 3. The same as in Fig. 2 for one selected coupled channel case with $t=1$. 
J. Golak et al.: A new way to perform partial wave decompositions of few-nucleon forces
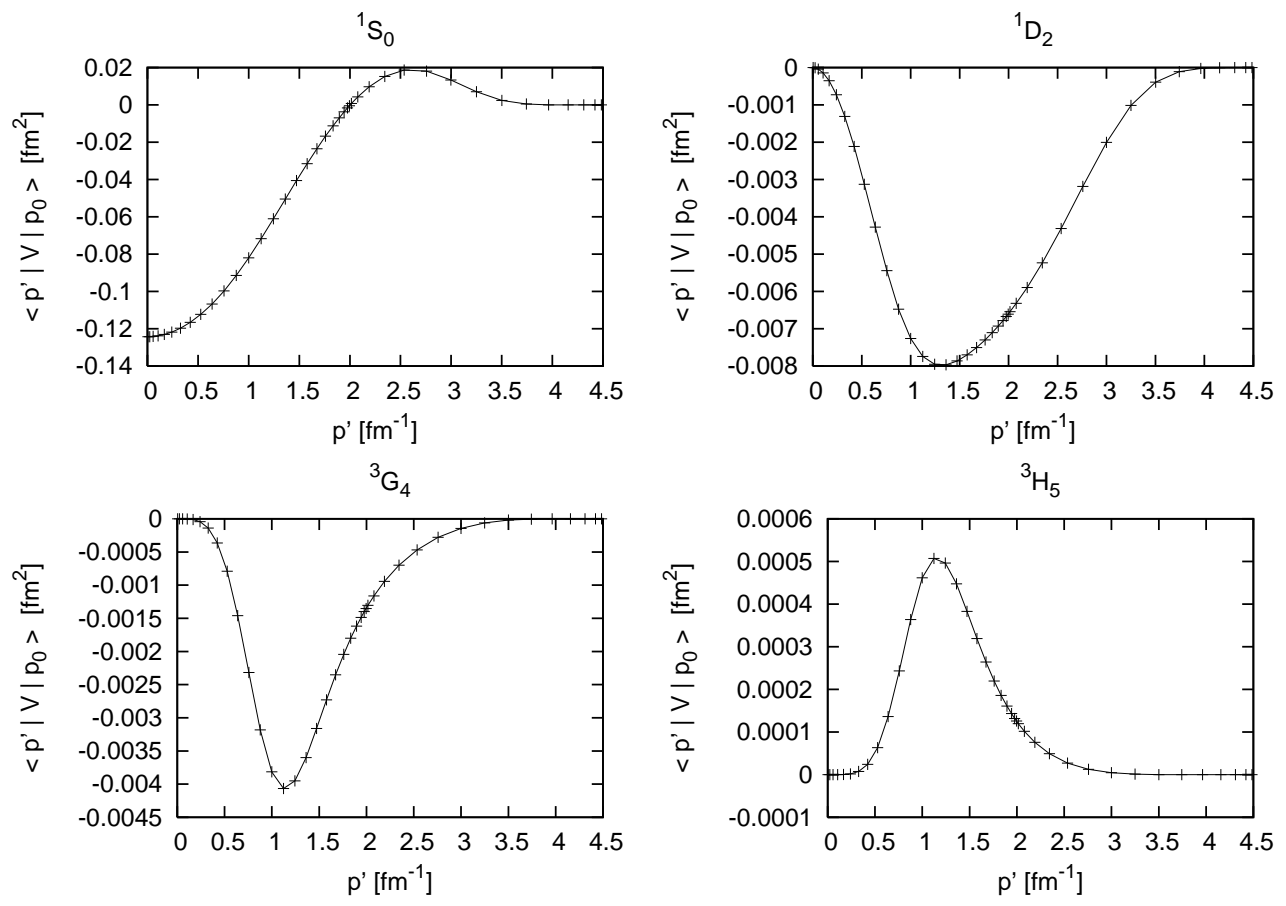

Fig. 4. The same as in Fig. 1 for the example of a chiral NNLO potential (see text for details).
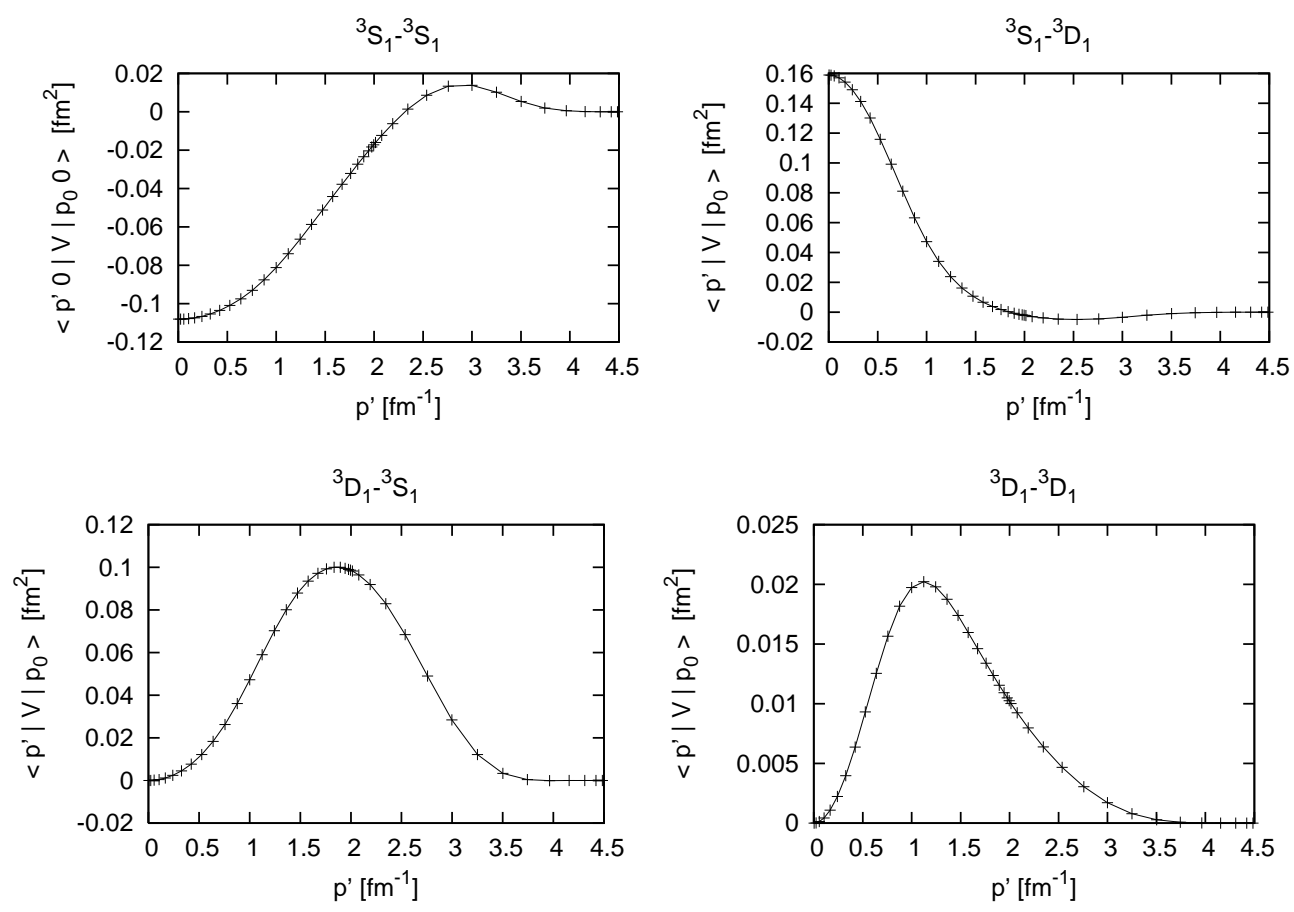

Fig. 5. The same as in Fig. 2 for the selected chiral NNLO potential. 

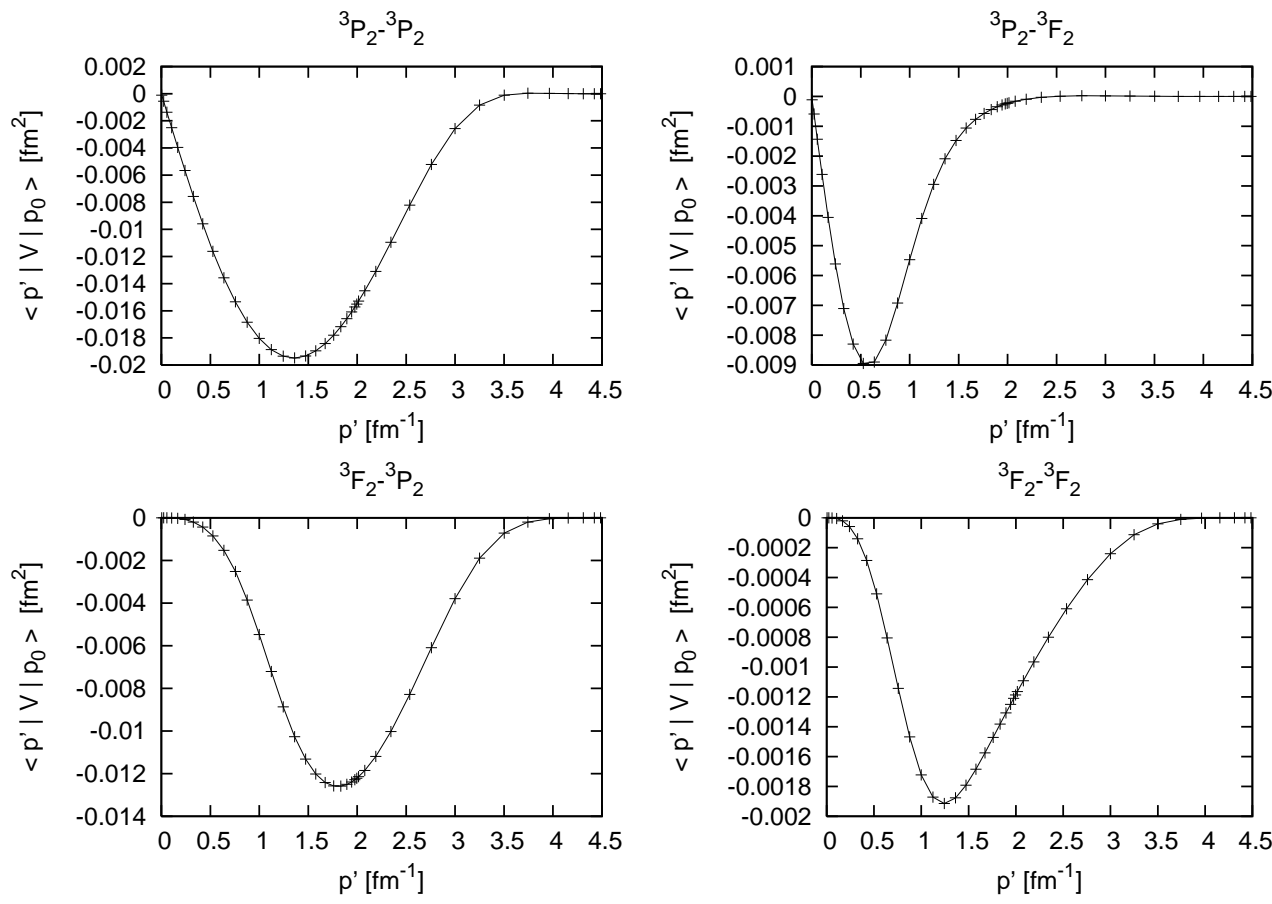

Fig. 6. The same as in Fig. 3 for the selected chiral NNLO potential. 